\begin{tabular}{lcr}
\hline \multicolumn{3}{c}{ A N N A L ES } \\
UNIVERSITATIS M A R I A E C URIE-SKŁ ODOWSK A \\
LOL. XXXVI & SECTIOFF & $1-2018$ \\
\hline
\end{tabular}

SEBASTIAN PIOTROWSKI

ORCID ID: https://orcid.org/0000-0002-4871-5131

Katolicki Uniwersytet Lubelski Jana Pawła II

RÉMY PORQUIER

ORCID ID: https://orcid.org/0000-0001-5105-0903

Université Paris Nanterre

\title{
Le chat à la fenêtre: l'expression de relations spatiales en polonais (L1) et en français (L2 et L1)
}

„Kot w oknie”: wyrażanie relacji przestrzennych w językach polskim (L1) i francuskim (L2 i L1)

"A cat in a window": Expressing spatial relations in Polish (L1) and French (L2 and L1)

\section{INTRODUCTION}

Cet article porte sur l'expression de relations spatiales dans deux langues, le français et le polonais; plus précisément sur l'expression des relations dans l'axe sagittal, exprimées de façon canonique en français par devant/derrière, et en polonais par przed/za.

Il s'inscrit dans le cadre sémantico-cognitif des recherches comparées sur l'expression dans les langues naturelles des relations spatiales, qui concernent à la fois la linguistique descriptive, la linguistique contrastive et l'acquisition des langues.

Est présenté d'abord (1) le cadrage théorique de la recherche: la place de ces relations dans les théories et les recherches sur l'expression des relations spatiales dans les langues (1.1); leur place dans les recherches sur l'acquisition des langues (1.2). Sont exposés ensuite (2) le dispositif d'enquête, avec sa portée, ses étapes et ses limites, puis (3) les résultats de l'enquête et leurs prolongements possibles. 


\section{CADRAGE THÉORIQUE}

1.1. L'expression des relations spatiales dans les langues naturelles implique trois composantes imbriquées : perceptuelle, sémantique et cognitive.

On y distingue les relations dynamiques, qui impliquent mouvement et/ou déplacement et les relations statiques (localisation, positionnement) qui impliquent divers modes de repérage. Nous nous intéressons ici à ces relations statiques: topologiques et projectives.

Une partie d'entre elles, les relations projectives (Piaget et Inhelder, 1947) s'inscrivent dans des coordonnées orthogonales correspondant à des paramètres anthropologiques, selon la position canonique de l'être humain dans l'espace, selon trois axes: vertical (haut/bas), latéral (gauche/droite), sagittal (devant/derrière), éventuellement cumulables (en haut à gauche, devant à droite, etc.).

Leur expression dans les langues repose sur deux repères de base:

- la relation entre la cible (objet à localiser) et le site (objet de référence) ${ }^{1}$, comme dans «la valise (cible) est devant la porte (site)»;

- l'origo ${ }^{2}$, c'est-à-dire la position, ou le point de vue, du locuteur ou de l'observateur par rapport à la configuration cible - site; ce peut aussi être le point de vue de l'interlocuteur, voire d'un tiers figuré ou translaté. L'origo est donc un point de vue qui se déplace.

Trois autres traits jouent un rôle dans l'expression des relations sagittales:

- l'orientation intrinsèque de la cible: un être humain, comme une voiture ou divers objets, a un avant et un arrière, une face et un dos;

- l'accès à la perception: la cible peut être totalement visible, non visible ou partiellement visible;

- le choix entre les perspectives (ou imageries) face-à-face et en tandem (Hill, $1991)^{3}$, pour situer la cible: dans la première perspective, l'orientation du locuteur reste fixe par rapport au site qui se trouve en face, dans la seconde, le locuteur transfère/impose son orientation à l'objet de référence (site).

Comme on le verra plus loin (2.1), ces distinctions sont toutes importantes dans la définition d'un protocole d'enquête.

1.2. L'acquisition des moyens d'expression linguistique des relations spatiales sur l'axe sagittal a fait l'objet de travaux:

- sur l'acquisition de L1 (Piaget et Inhelder, 1947; Piérart, 1977; Slobin, 1993 ; Verjat, 1991 ; Robin, 2002),

${ }^{1}$ Les termes cible - site sont de Vandeloise (1986).

${ }^{2}$ Le terme et la notion d'origo ont été introduits par Bühler en 1934 (Bühler, 2004).

${ }^{3}$ Cette distinction est particulièrement saillante dans le cas de la relation latérale gauche droite: dans une relation en face-à-face, ce qui est à ma gauche est à droite pour mon vis-à-vis. 
- sur l'acquisition des langues secondes ou étrangères (Becker et Carroll, 1997; Hendriks, 1998; Hirzalla, 2009).

Quant à leur ordre d'acquisition, dans des L1 diverses et des L2, il a été observé (Carroll, 1992; Slobin, 1993) que, de façon générale ou tendancielle,

- l'expression des relations topologiques est acquise avant celle des relations projectives,

- pour les relations projectives, l'ordre d'acquisition serait:

$$
\text { axe vertical } \rightarrow \text { axe latéral } \quad \rightarrow \quad \text { axe sagittal }
$$

ce qui suggère que la maîtrise des relations sur l'axe sagittal serait cognitivement plus complexe $\mathrm{e}^{4}$. Les moyens linguistiques d'expression de telles relations varient selon les langues, mais, pour une partie des langues déjà explorées, sont de type lexical: pré- ou post-positions ou adverbes.

\section{DISPOSITIF DE LA TÂCHE}

\subsection{La démarche}

Le support est une série de 8 images (1 à 8$)$, présentant un chat de part et d'autre d'une fenêtre.
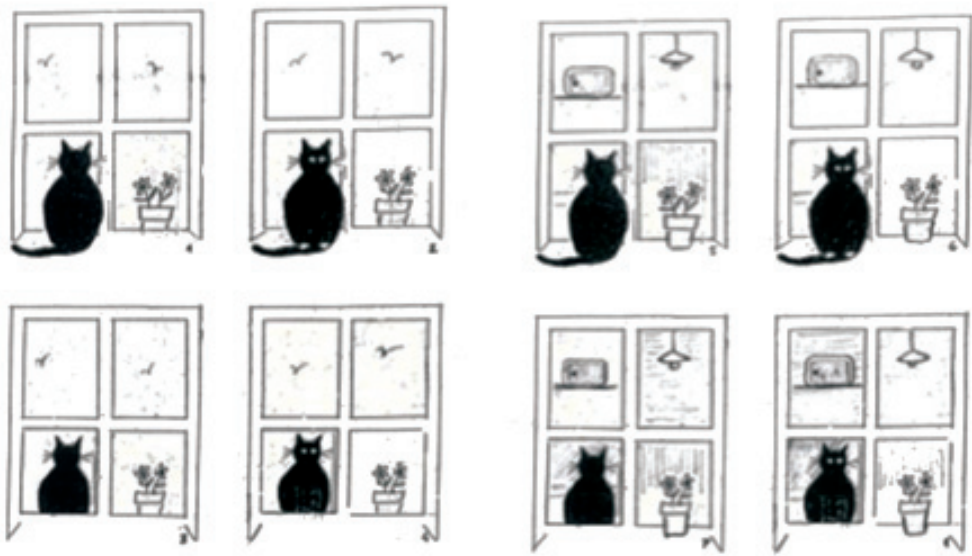

4 A noter que c'est la stabilité de l'axe vertical (l'orientation haut/bas reste constante malgré le déplacement de l'être humain), qui favorise l'acquisition des formes d'expression des relations le concernant avant celles référant aux axes latéral et sagittal, qui s'avèrent nettement plus complexes à maîtriser (Carroll, 1992). 
L'agencement des 8 images déploie et recoupe plusieurs paramètres, selon lesquels :

- le chat est à l'intérieur ou à l'extérieur de la maison,

- le chat est vu de l'intérieur ou de l'extérieur de la maison,

- le chat est vu de face ou de dos, ce qui fournit 8 configurations :

\begin{tabular}{|c|c|c|c|}
\hline $\begin{array}{c}\text { IMAGE 1 } \\
\text { chat à l'intérieur } \\
\text { vu de dos } \\
\text { vu de l'intérieur }\end{array}$ & $\begin{array}{c}\text { IMAGE 2 } \\
\text { chat à l'intérieur } \\
\text { vu de face } \\
\text { vu de l'intérieur }\end{array}$ & $\begin{array}{c}\text { IMAGE 5 } \\
\text { chat à l'extérieur } \\
\text { vu de dos } \\
\text { vu de l'extérieur }\end{array}$ & $\begin{array}{c}\text { IMAGE 6 } \\
\text { chat à l'extérieur } \\
\text { vu de face } \\
\text { vu de l'extérieur }\end{array}$ \\
\hline $\begin{array}{c}\text { IMAGE 3 } \\
\text { chat à l'extérieur } \\
\text { vu de dos } \\
\text { vu de l'intérieur }\end{array}$ & $\begin{array}{c}\text { IMAGE 4 } \\
\text { chat à l'extérieur } \\
\text { vu de face } \\
\text { vu de l'intérieur }\end{array}$ & $\begin{array}{c}\text { IMAGE 7 } \\
\text { chat à l'intérieur } \\
\text { vu de dos } \\
\text { vu de l'extérieur }\end{array}$ & $\begin{array}{c}\text { IMAGE 8 } \\
\text { chat à l'intérieur } \\
\text { vu de face } \\
\text { vu de l'extérieur }\end{array}$ \\
\hline
\end{tabular}

Dans la conception initiale puis dans le choix final des 8 images, ont ainsi été pris en compte:

- l'axe sagittal observateur - chat, constant dans les 8 images,

- la position statique de la cible (le chat) ${ }^{5}$,

- le changement de position de la cible (chat dedans/dehors),

- le changement de position de l'observateur (dedans/dehors),

- l'orientation intrinsèque : c'est parce que le chat a un avant et un arrière qu'il peut être vu de face ou de dos.

Quant à l'accès à la perception, le choix de la fenêtre comme site permet d'une part, par sa transparence, de voir l'orientation du chat - par rapport à l'observateur et par rapport à la fenêtre - et d'autre part, par son encadrement, qui dans 4 images produit une occultation très partielle de la cible, de percevoir de quel côté de la vitre se trouve le chat.

2.2. Il s'agit, pour l'informateur-observateur (en position d'origo), de répondre à la question: «où est le chat par rapport à la fenêtre?». Pour ce qui est de la consigne, il est à souligner que l'enquêteur avait informé les informateurs que les seules réponses admises étaient devant/derrière, pour le français, et przed/za, pour le polonais.

Les huit images ont été présentées, une par une, collectivement, en deux phases:

5 Dans sa propre recherche menée sur 4 images, Hirzalla (2009) prend en compte la perception et la verbalisation du déplacement du chat (comme s'il bougeait d'une image à l'autre), en termes de mouvement référentiel. 
- à 20 étudiants polonais ( $3^{\mathrm{e}}$ année de philologie romane) en polonais, réponse en polonais,

- aux mêmes étudiants polonais (une semaine après) en français, réponse en français.

Sur les 20 informateurs, initiaux, seuls 12 ont pu être retenus pour la comparaison des réponses individuelles en français et en polonais (au total 192 unitésréponses).

Les huit images ont été plus tard soumises à 4 autres informateurs, 2 polonophones natifs et 2 francophones natifs, avec confrontations sur leurs verbalisations des images $3,4,5,8$.

2.3. On a pu noter, in vivo et a posteriori, quelques biais quant au principe et au déroulement de la tâche:

- l'effet test: présomption d'une bonne réponse à trouver (et éventuellement de 8 réponses différentes pour les 8 images),

- la succession (et le mode de présentation) relativement rapide des 8 images, de nature à perturber la décision renouvelée de choix verbal,

- certaines difficultés de lecture de l'image: distinction entre le dedans et le dehors, entre le chat de face et de dos.

\section{RÉSULTATS}

\subsection{Le recueil}

Le recueil de réponses auprès des 16 informateurs retenus pour l'étude (12 étudiants, 2 francophones natifs et 2 polonophones natifs) fournit, pour 8 images et 2 langues, 224 unités-réponses, présentées dans le tableau ci-dessous (page 130).

Un certain nombre de réponses, inexploitables ou non pertinentes, n'ont pas été prises en compte:

- POL : tyłem, przodem, z drugiej strony, po lewej stronie, naprzeciwko, etc.

- FR: en face, à côté, en bas, au-dessus, en haut, après, etc.

On constate, chez les étudiants polonophones, une grande hétérogénéité des réponses par langue, par individu et par image:

- par langue

FR: 21 réponses différentes (moyenne 2,65 par image)

POL : 30 réponses différentes (moyenne 3,75 par image)

- par individu

FR: entre 2 (informateur E12) et 7 (informateur E8) (moyenne 4,25)

POL : entre 2 (informateur E10) et 8 (informateur E9) (moyenne 5,83) 


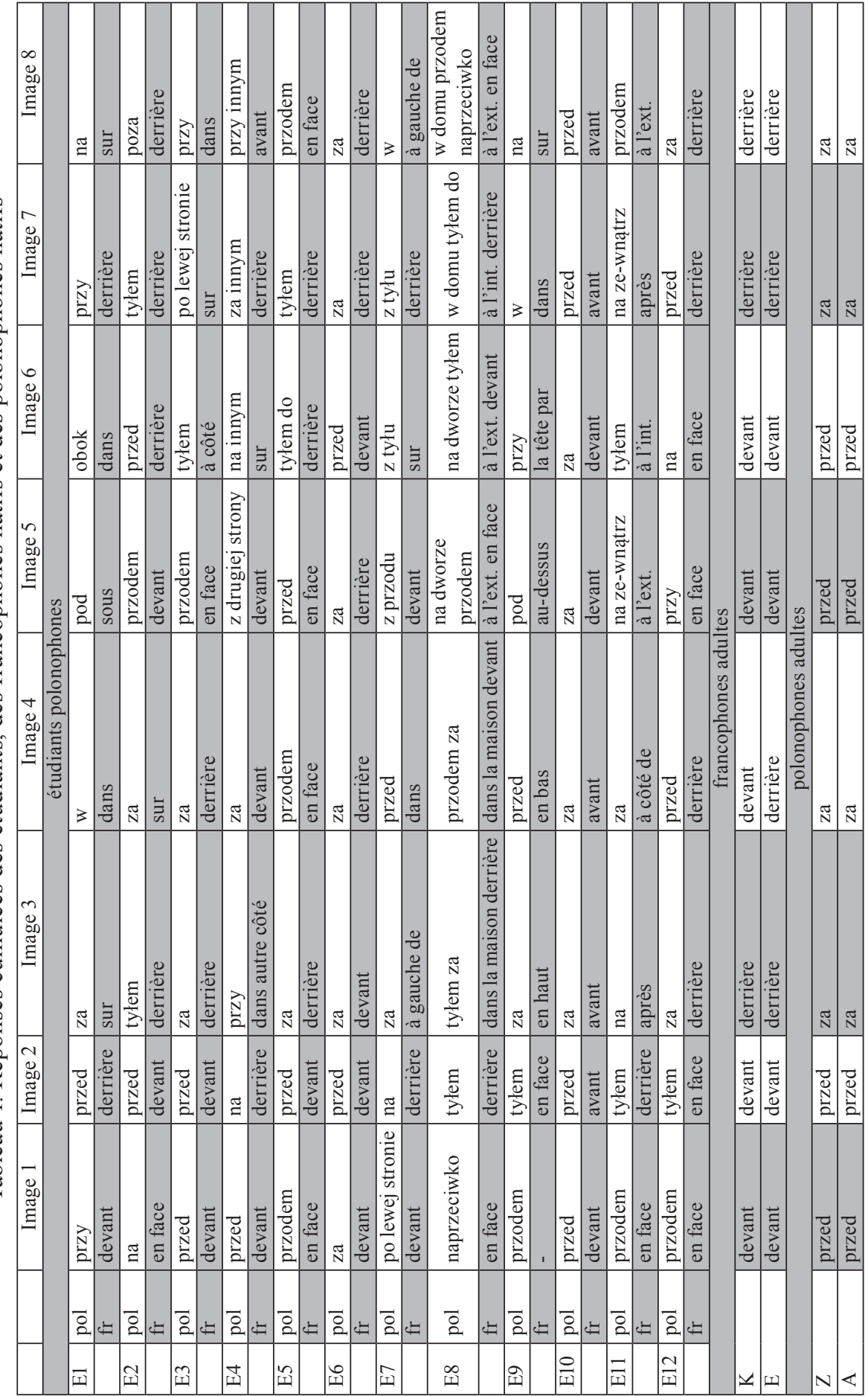


- par image

FR: entre 2 (image 1) et 9 (image 4) (moyenne 6,25)

POL: entre 3 (image 2) et 10 (image 7) (moyenne 7,75)

On observe également un nombre important de réponses composées, analytiques, qui verbalisent deux ou trois des paramètres de l'image:

- FR: dans la maison derrière, dans la maison devant, à l'extérieur en face, à l'extérieur devant, à l'intérieur derrière, à l'extérieur en face (E8)

- POL: naprzeciwko, tytem, tytem za, na dworze tytem, przodem za, na dworze przodem, $w$ domu tytem do, $w$ domu przodem naprzeciwko $(\mathrm{E} 8)^{6}$.

\subsection{Réponses en przed/za et devant/derrière}

Si l'on observe, chez les mêmes 12 étudiants polonophones, le total des réponses en $\mathrm{przed} / \mathrm{za}$ et devant/derrière et leurs éventuels couplages entre les deux langues ${ }^{7}$, on constate que leur total se monte à 80 :

przed (16) $\quad z a(22)$

devant (18) derrière (24)

et que leur distribution, par couplages individuels bilingues, est la suivante:

Tableau 2. Couplages FR + POL chez les étudiants

\begin{tabular}{|l|c|c|c|}
\hline & Przed & Za & Autres \\
\hline Devant & 8 & 5 & 5 \\
\hline Derrière & 3 & 9 & 12 \\
\hline Autres & 5 & 8 & 41 \\
\hline
\end{tabular}

Sur le total de 96 couples FR+POL, on obtient seulement 17 couples de réponses homogènes (couples devant + przed et derrière $+z a$, marqués en gris dans le tableau). Les réponses apparaissent donc hétérogènes jusqu'à manifester, dans certains profils individuels, de possibles incohérences ou contradictions, peu imputables à une maîtrise insuffisante du français.

À ce stade, une seule tendance, à la fois intralinguistique et interlinguistique, se laisse observer: les informateurs suivent dans la majorité des cas une règle implicite référant à l'accès à la perception. Selon Vandeloise (1986), la distinction devant/derrière obéit à deux régimes différents : l'un est lié à l'orientation générale du site, l'autre à l'accès à la perception. Dans le second cas, le choix de derrière est

${ }^{6}$ Il s'agit là, en français et en polonais du même informateur E8.

${ }^{7}$ Il n'est pas utile de préciser ici que le but de l'expérience n'était pas d'apprécier, en forme de test ou d'évaluation, les compétences des étudiants-informateurs, même si leurs propres représentations ont pu avoir une influence sur les résultats. 
dicté par la dissimulation de la cible: le locuteur aurait tendance à situer la cible derrière le site à chaque fois qu'elle serait partiellement cachée, que le site soit intrinsèquement orienté ou pas.

Dans les tableaux ci-dessous, on présente les réponses se rapportant respectivement aux images 1, 2, 5, 6 (le chat n'y est pas caché) et aux images 3, 4, 7, 8 (le chat y est partiellement caché).

Tableau 3 . Choix des informateurs pour cible non cachée

\begin{tabular}{|l|c|c|c|c|c|c|}
\hline & Choix & Réponses $^{8}$ & Choix & Réponses & Total devant & Total derrière \\
\hline \multirow{2}{*}{ Image 1 } & devant & $6+2$ & przed & $3+2$ & 13 & 1 \\
\cline { 2 - 8 } & derrière & 0 & za & 1 & & \\
\hline \multirow{2}{*}{ Image 2 } & devant & $4+2$ & przed & $6+2$ & 14 & 5 \\
\cline { 2 - 7 } & derrière & 5 & za & 0 & & \\
\hline \multirow{2}{*}{ Image 5 } & devant & $4+2$ & przed & $1+2$ & 9 & 3 \\
\cline { 2 - 8 } & derrière & 1 & za & 2 & & 3 \\
\hline \multirow{2}{*}{ Image 6 } & devant & $2+2$ & przed & $2+2$ & 8 & \\
\cline { 2 - 7 } & derrière & 2 & za & 1 & & \\
\hline
\end{tabular}

Tableau 4. Choix des informateurs pour cible cachée

\begin{tabular}{|l|c|c|c|c|c|c|}
\hline & Choix & Réponses & Choix & Réponses & Total devant & Total derrière \\
\hline \multirow{2}{*}{ Image 3 } & devant & 1 & przed & 0 & 1 & 16 \\
\cline { 2 - 7 } & derrière & $4+2$ & za & $8+2$ & & \\
\hline \multirow{2}{*}{ Image 4 } & devant & $1+1$ & przed & 3 & 5 & 12 \\
\cline { 2 - 7 } & derrière & $3+1$ & za & $6+2$ & & \\
\hline \multirow{2}{*}{ Image 7 } & devant & 0 & przed & 2 & 2 & 12 \\
\cline { 2 - 7 } & derrière & $7+2$ & za & $1+2$ & & 9 \\
\hline \multirow{2}{*}{ Image 8 } & devant & 0 & przed & 1 & 1 & \\
\cline { 2 - 7 } & derrière & $3+2$ & za & $2+2$ & & \\
\hline
\end{tabular}

$\mathrm{Au}$ vu des résultats, on peut constater que les informateurs choisissent massivement devant lorsque la cible n'est pas dissimulée (tableau 3), et que, inversement, ils choisissent très majoritairement derrière lorsque la cible est dissimulée (tableau 4). Il est intéressant d'observer que la règle en jeu (selon laquelle derrière signale l'absence de visibilité) reste pertinente même dans le cas où la visibilité n'est que très peu brouillée (ici, seules les pattes et la queue du chat sont cachées).

Ajoutons que lorsque les informateurs choisissent devant pour cible non cachée ou derrière pour cible cachée, ils adoptent une perspective déictique et l'imagerie

${ }^{8}$ Les réponses cumulent les choix des étudiants (en première position) et des adultes (en deuxième position). Par exemple «devant» et « $6+2 »$ signifie que 6 étudiants et deux adultes francophones ont fait ce choix. Respectivement, «przed» et « $3+2 »$ signifie que 3 étudiants et deux adultes polonophones ont fait ce choix. 
face-à-face. Et lorsqu'ils choisissent derrière pour cible non cachée ou devant pour cible cachée, ils adoptent une perspective non déictique et l'imagerie en tandem. En dehors de l'image 4, où les choix des informateurs se répartissent de façon plus équilibrée, l'imagerie en tandem reste donc largement minoritaire.

$\mathrm{Si}$, en théorie, les deux types d'imagerie concernent la relation entre le locuteur (origo) et le monde extérieur (site) et ne prennent pas en compte la cible, ni son orientation, ni ses propriétés, il semble bien que l'élément cible ait joué un certain rôle dans les choix faits par nos informateurs. Si l'on compare les images 3 et 4, qui présentent toutes les deux le chat à l'extérieur, vu de l'intérieur, on constate que le changement de perspective (passage de face-à-face à en tandem) s'opère presque exclusivement dans le cas de l'image 4 , c'est-à-dire là où le chat est vu de face. Comme l'a dit (à propos de l'image 4) un des informateurs lors de l'autoconfrontation, «le chat regarde vers l'intérieur [...], ça veut dire qu'il a envie de rentrer». Cette clarification montre aussi que c'est l'aspect intérieurlextérieur qui a joué un rôle dans son choix, et pas uniquement l'aspect faceldos. Cela explique pourquoi le changement de perspective ne s'opère pas dans le cas de l'image 8 , où le chat est aussi vu de face, mais se trouve bien installé à l'intérieur. Dans ce caslà, les informateurs ont très majoritairement opté pour derrière, tout en adoptant l'imagerie face-à-face.

\subsection{Les commentaires en autoconfrontation}

La phase deux de l'enquête a consisté à solliciter les réponses puis les commentaires de deux adultes francophones (E, K) et deux adultes polonophones $(\mathrm{A}, \mathrm{Z})$. Leurs commentaires portent sur les réponses qu'ils avaient données à propos des images 3, 4, 5 et 8, images jugées le plus à même de susciter un changement de perspective. Il en ressort explicitement que les choix des informateurs pour telle ou telle expression (devant $v s$. derrière/przed $v s$. za) dépendent de plusieurs facteurs comme

- construction de la scène (intérieur/extérieur, présence d'autres objets): «le chat est à l'intérieur, car il se trouve du même côté que la radio et la lampe, alors que les fleurs sont à l'extérieur» (A), «le chat est à l'intérieur de la maison et il regarde dehors, il est devant» (E),

- construction du site (transparence, propriétés techniques):

«le dormant de la fenêtre suggère où se trouve l'intérieur» $(Z)$, «le chat se trouve à l'intérieur, et dans les vitres se reflètent les objets de la pièce» $(Z)$, «il cache un peu un des carreaux de la fenêtre» (E),

- propriétés de la cible (aspect animé, potentiellement dynamique, orientation): 
«il est à l'extérieur, et il ne cherche pas à rentrer, il regarde vers l'extérieur» $(\mathrm{K})$, «le chat regarde vers l'intérieur, ça veut dire qu'il a envie de rentrer» $(\mathrm{K})$,

- position de l'origo (accès à la perception, présence d'obstacle) :

«il est à l'extérieur, il est un peu caché par la fenêtre» (E), «il est entre nous et la fenêtre, il est devant» (K).

Si tous ces facteurs influencent les choix des informateurs, quelle que soit la langue d'expression, on remarque aussi certaines tendances ${ }^{9}$ :

- les polonophones adultes attachent une plus grande importance à l'aspect technique de la scène et à la présence des objets autres que la cible,

- les francophones adultes attachent une plus grande importance à l'aspect animé de la cible,

- aussi bien les polonophones que les francophones attachent une grande importance à la construction de la scène et confirment la règle «le manque de visibilité appelle l'emploi de derrière».

\section{CONCLUSIONS}

Quant aux résultats, il ressort des analyses que:

- les choix entre devant/derrière et przed/za ne suffisent pas à décrire les images; malgré une consigne contraignante, les informateurs ont démontré une nette tendance à recourir à des formulations composées, parfois ne faisant pas référence aux prépositions attendues dans le cadre de l'enquête; les précisions apportées ont concerné la perception du chat (de face / de dos), la position de l'origo (de l'intérieur / de l'extérieur) ou la localisation du chat (dans/ hors de la maison);

- le choix entre les imageries face-à-face et en tandem, mettant en jeu la relation origo - site, s'opère en fonction de plusieurs paramètres comme: construction de la scène, propriétés de la cible, accès à la perception;

- la règle de Vandeloise (1986), quant à l'emploi de derrière là où la cible est partiellement dissimulée, s'avère pertinente aussi bien pour le français que pour le polonais.

Le déroulement de notre enquête appelle plusieurs remarques d'ordre méthodologique, notamment quant à la conception du protocole et des modalités de recueil de données. Il est à envisager de signaler aux informateurs la possibilité de deux réponses pour certaines images. Le déroulement de la tâche peut également être envisagé différemment, par exemple en demandant aux informateurs non de

\footnotetext{
${ }^{9}$ Ces tendances, enregistrées auprès de 4 sujets, ne sont en aucun cas généralisables.
} 
localiser le chat dans des images, mais de l'y placer selon des indications orales. D'autre part, l'apport des autoconfrontations apparaît nécessaire, en ce qu'il permet d'interpréter les réponses et de vérifier, à travers les différences individuelles, le rôle de l'imagerie face-à-face / en tandem.

Les dimensions du corpus recueilli apparaissent trop limitées pour envisager, compte tenu de la diversité des paramètres, une analyse multifactorielle à fiabilité statistique. Mais en pareil cas, une analyse qualitative individuelle et interindividuelle est nécessaire, informée par des commentaires en autoconfrontation.

L'expérience présentée, portant sur le polonais L1 et le français L2 (et, complémentairement, sur le français L1) suggère une extension à diverses langues et à divers couples de L1-L2, impliquant des langues typologiquement différentes.

Quant au polonais, on entrevoit l'intérêt d'enquêtes sur le polonais L2 auprès de sujets de L1 diverses, mais aussi de leur contribution à une description détaillée (à l'aide et au-delà des travaux de Cienki, 1989; Okoniowa, 1994; Dancygier, 1997 ; Bacz, 2003; Tabakowska, 2003; Kopecka, 2004) de l'expression de la relation sagittale en polonais.

\section{RÉFÉRENCES BIBLIOGRAPHIQUES}

Bacz, B. (2003). A preliminary semantic analysis of the Polish preposition. Langues et linguistique, $29,1-23$.

Becker, A., Carroll, M. (eds.). (1997). The acquisition of spatial relations in a second language, Amsterdam: John Benjamins.

Bühler, K. (2004). Teoria języka. Trad. J. Koźbiał. Kraków: Universitas.

Carroll, M. (1992). Comment s'organise-t-on pour parvenir à acquérir? Ce que nous enseigne la performance de l'adulte apprenant une deuxième langue. Acquisition et interaction en langue étrangère, 1, 37-51.

Cienki, A. (1989). Spatial cognition and the semantics of prepositions in English, Polish, and Russian. Slavistische Beiträge, 237, 1-172.

Dancygier, B. (1997). How Polish structures space. Prepositions, direction nouns, case, and metaphor. In: A. Follen, F. Van Der Leek (eds.), Constructions in cognitive linguistics (pp. 27-45). Amsterdam: John Benjamins.

Hendriks, H. (1998). Comment il monte le chat? en grimpant! Acquisition et interaction en langue étrangère, 11, 147-190.

Hill, C. (1991). Recherches interlinguistiques en orientatation spatiale. Communications, 53(1), 171-207.

Hirzalla, A. (2009). Where is the cat in relation to the window? The expression of spatial relations in French and Arabic first and second language. California Linguistic Notes, 34(2), 1-31.

Kopecka, A. (2004). Etude typologique de l'expression de l'espace: localisation et déplacement en français et en polonais. Thèse, Université Lyon 2.

Okoniowa, J. (1994). Przeciwstawienia kierunkowe w języku polskim. Kraków: Polska Akademia Nauk.

Piaget, J., Inhelder, B. (1947). La représentation de l'espace chez l'enfant. Paris : PUF. 
Piérart, B. (1977). L'acquisition du sens des marqueurs de relation spatiale devant et derrière. L'année psychologique, 77(1), 95-116.

Robin, F. (2002). Production et coordination des termes spatiaux entre 6 et 9 ans. Enfance, 54(4), 363-379. Retrieved from http://www.cairn.info/revue-enfance-2002-4-p-363.htm (11.02.2018).

Slobin, D. (1993). Adult language acquisition: a view from child language study. In: C. Perdue (ed.), Adult language acquisition: cross-linguistic perspectives. Vol. II (pp. 239-252). Cambridge: Cambridge University Press.

Tabakowska, E. (2003). Space and time in Polish: The preposition $z a$ and the verbal prefix $z a-$. In: H. Cuyckens, T. Berg, R. Dirven, K. Panther (eds.), Motivation in language, Studies in honor of Günter Radden (pp. 153-177). Amsterdam: John Benjamins.

Vandeloise, C. (1986). L'espace en français. Sémantique des prépositions spatiales. Paris: Seuil.

Verjat, I. (1991). Le statut cognitif des marqueurs devant, derrière chez l'enfant français. L'année psychologique, 91(2), 207-229.

\section{RÉSUMÉ}

Cet article, qui s'inscrit dans le cadre des recherches interlinguistiques, porte sur un domaine précis de la référence spatiale: la référence projective sur l'axe sagittal ('devant'/'derrière', 'przed'/'za'), qui appelle non seulement l'acquisition d'un lexique spécifique mais également, du point de vue cognitif, l'appropriation de distinctions sémantiques liées à la perception et aux représentations de l'espace.

La démarche empirique adoptée, de forme semi-expérimentale, a consisté à recueillir les verbalisations, en polonais (L1) et en français (L2 et L1), de 20 jeunes adultes, à partir d'une série d'images regroupant diverses configurations spatiales statiques. Ce corpus est complété pour comparaison par les productions, sur les mêmes images, de francophones et de polonophones natifs.

L'analyse des résultats vise à alimenter et à développer, pour le domaine concerné, les descriptions existantes du polonais, à questionner les descriptions antérieures proposées pour le français et à affiner les comparaisons polonais - français existantes.

Mots-clés : relations spatiales, référence projective, acquisition, comparaison polonais-français

\section{ABSTRACT}

The paper presents a study on the expression of spatial reference in Polish and French from the perspective of acquisition of French by Polish language speakers. Our study deals with the problem of spatial reference: projective reference on sagittal axis (devant/derrière, przed/za), which refers not only to the acquisition of specific lexical items but, what is equally important from the cognitive point of view, to the appropriation of semantic distinctions related to the perception and representation of space.

The empirical approach adopted in the study, in a semi-experimental manner, consisted in collecting utterances, in Polish (L1) and French (L2 and L1), of 20 young adults, concerning a series of pictures presenting various static spatial configurations. The corpus was then complemented, for comparison purposes, by French and Polish native speakers' output in reaction to the same pictures.

The aim of the analysis is to propel and add new elements to the existing descriptions of the Polish language, to approach from a different perspective the descriptions previously provided for French, and make a contribution to Polish-French comparative studies. studies

Keywords: spatial relations, projective reference, acquisition, Polish-French comparative 


\begin{abstract}
ABSTRAKT
Przedmiotem artykułu jest sposób wyrażania relacji przestrzennych w językach polskim i francuskim w procesie akwizycji języka francuskiego przez polonofonów. Badanie odnosi się do konkretnego wycinka owych relacji: wyrażania umiejscowienia obiektu w osi strzałkowej (devant/ derrière, przed/za), które wymaga od uczących się języka nie tylko opanowania określonego słownictwa, ale również - z kognitywnego punktu widzenia - przyswojenia opozycji semantycznych związanych z reprezentacją przestrzeni.

Badanie polegało na przedstawieniu w języku polskim (L1) i francuskim (L2 i L1) serii obrazków ukazujących różne statyczne konfiguracje przestrzenne. Uczestnikami badania byli studenci filologii romańskiej (20 osób), dwoje dorosłych polonofonów i dwoje dorosłych frankofonów.

Zebrany materiał stanowi skromny wkład do opisu akwizycji języka francuskiego przez polonofonów, do opisu języka polskiego, a także do polsko-francuskich badań kontrastywnych.
\end{abstract}

Słowa kluczowe: akwizycja języka obcego, relacje przestrzenne, polsko-francuskie badania kontrastywne 
